\title{
The Banana Distribution is Gaussian: A Localization Study with Exponential Coordinates
}

\author{
Andrew W. Long *, Kevin C. Wolfe ${ }^{\dagger}$, Michael J. Mashner ${ }^{\dagger}$, Gregory S. Chirikjian ${ }^{\dagger}$ \\ * Northwestern University, Evanston, IL 60208 \\ $\dagger$ Johns Hopkins University, Baltimore, MD 21218
}

\begin{abstract}
Distributions in position and orientation are central to many problems in robot localization. To increase efficiency, a majority of algorithms for planar mobile robots use Gaussians defined on positional Cartesian coordinates and heading. However, the distribution of poses for a noisy two-wheeled robot moving in the plane has been observed by many to be a "bananashaped" distribution, which is clearly not Gaussian/normal in these coordinates. As uncertainty increases, many localization algorithms therefore become "inconsistent" due to the normality assumption breaking down. We observe that this is because the combination of Cartesian coordinates and heading is not the most appropriate set of coordinates to use, and that the banana distribution can be described in closed form as a Gaussian in an alternative set of coordinates via the so-called exponential map.

With this formulation, we can derive closed-form expressions for propagating the mean and covariance of the Gaussian in these exponential coordinates for a differential-drive car moving along a trajectory constructed from sections of straight segments and arcs of constant curvature. In addition, we detail how to fuse two or more Gaussians in exponential coordinates together with given relative pose measurements between robots moving in formation. These propagation and fusion formulas utilized here reduce uncertainty in localization better than when using traditional methods. We demonstrate with numerical examples dramatic improvements in the estimated pose of three robots moving in formation when compared to classical Cartesiancoordinate-based Gaussian fusion methods.
\end{abstract}

\section{INTRODUCTION}

A rich area of robotics research known as SLAM or simultaneous localization and mapping consists of a robot mapping its environment while estimating where it may be in this map. To incorporate uncertainty, one strategy represents all possible poses of the robot with a probability density function (pdf). The goal then is to maintain and update this pdf as the robot moves. One solution is to propagate the entire pdf such as with Fourier transform techniques [26]. However, these techniques can be too numerically intensive for real-time SLAM applications.

According to Durrant-Whyte and Bailey, the two most common tools used to increase efficiency for SLAM algorithms are the extended Kalman filter (EKF) and the Rao-Blackwellized (RB) particle filter [8]. EKF-SLAM is based on the classic work of Smith et al. in which linearized models are used to propagate uncertainty of the non-linear motion and observations [21]. Several strategies for improving the efficiency for large scale SLAM problems using this technique are documented by Bailey and Durrant-Whyte [2]. By using the RB particle filter, Murphy observed that the SLAM problem

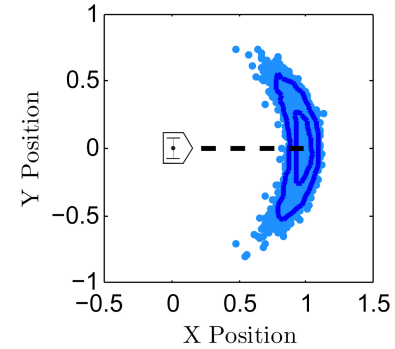

(a)

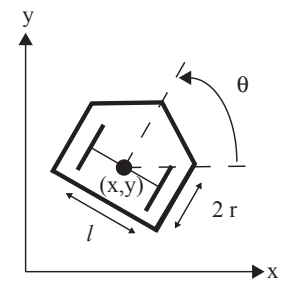

(b)
Fig. 1. (a) Banana-shaped distribution for position of a differential-drive robot moving along a straight line with noisy wheel speeds. (b) Notation for differential-drive robot.

could be decomposed in to a robot localization problem and several independent landmark estimation problems [18]. With this factorization, many efficient SLAM algorithms known as FastSLAM have been proposed [16, 17, 11].

An inherent technique utilized in EKF-SLAM and many FastSLAM algorithms for robots operating in the plane is to represent all distributions with Gaussians in Cartesian coordinates $(x, y)$ and orientation heading angle $(\theta)$. This Gaussian/normal representation can be fully parameterized by a mean and covariance in these variables. Equal probability density contours of these distributions are described by ellipses. However, if we command a differential-drive robot to drive along a straight path, the resulting distribution from many sample paths may look similar to Fig. 1(a). Originally described by Thrun et al. [23], [24], this distribution is generally referred to as the "banana-shaped" distribution, which does not have elliptical probability density contours in the variables $(x, y, \theta)$. As the uncertainty grows, this assumption of normality breaks down and the maps inevitably become inconsistent [14].

Many have studied the problem of inconsistency for the EKF-SLAM [14], [3], [5], [13], and [6]. Julier and Ulhmann proved that this inconsistency is a direct result of linearization of the non-linear models in EKF-SLAM [14]. The extent of the inconsistency was studied by Bailey et al. in the context of heading uncertainty. They showed that if the standard deviation on the heading was larger than one or two degrees the map ultimately failed, specically with regards to excessive information gain and jagged vehicle trajectories [3]. In addition, the quality of the normal assumption in these coordinates was 
studied for the RB particle filter in [22], which demonstrated that several real-world examples were not well described by Gaussians.

In the majority of existing algorithms, there is an inherent assumption that the distribution should be represented by Gaussians in Cartesian coordinates. In this paper, we propose to use exponential coordinates and Lie groups to represent the robot's pose. We demonstrate that a Gaussian in these coordinates for planar robots provides a much better fit than a Gaussian in Cartesian coordinates as the uncertainty increases. By using this approach, we can derive closed-form propagation and fusion formulas that can be used to estimate the mean and covariance of the robot's pose.

In the last few years, the idea of using the Lie group nature of rigid body transformations has drawn some attention for the SLAM problem, particularly in the area of monocular camera SLAM [15], [20], [10]. General robot motion utilizing rigid body transformations has been applied with Consistent Pose Registration (CPR) [1] and complementary filters [4].

The outline of the paper is as follows. In Section III. we derive the stochastic differential equation for the differentialdrive robot operating in the plane. We review rigid body motions and their relationship to exponential coordinates in Section III] We provide the definitions for the mean, covariance and the Gaussian probability density function in these coordinates in Section IV] A comparison between the Gaussian in Cartesian coordinates and the Gaussian in exponential coordinates is detailed in Section V] In Section VI, we derive closed-form expressions that can be used to propagate the mean and covariance of the Gaussian in exponential coordinates. We conclude by deriving closed-form formulas for fusing two (or more) Gaussians in exponential coordinates in Section VII These fusion formulas are then applied to multiple robots moving in formation.

\section{Stochastic Differential Equation}

The example we will be considering in this paper is the kinematic cart or differential-drive robot. This robot has two wheels each of radius $r$ which can roll but not slip. The two wheels share an axis of rotation and are separated by a length $\ell$. The configuration of the robot can be represented as $\mathbf{x}=$ $[x, y, \theta]^{T}$ where $(x, y)$ is the Cartesian position in the plane of the center of the axle and $\theta$ is the orientation of the robot as shown in Fig. 1(b) For the configuration coordinates $\mathbf{x}$, the governing differential equations are

$$
\begin{gathered}
d x=\frac{r \cos \theta\left(d \phi_{1}+d \phi_{2}\right)}{2}, \quad d y=\frac{r \sin \theta\left(d \phi_{1}+d \phi_{2}\right)}{2}, \\
\text { and } d \theta=\frac{r}{\ell}\left(d \phi_{1}-d \phi_{2}\right),
\end{gathered}
$$

where the rate of spinning of each wheel is given by $\omega_{i}=$ $d \phi_{i} / d t$ for $i=1$ or 2 . If the wheel speeds are governed by stochastic differential equations (SDE) as

$$
d \phi_{i}=\omega_{i}(t) d t+\sqrt{D} d w_{i} \quad \text { for } i=1 \text { or } 2
$$

where $d w_{i}$ are unit-strength Wiener processes and $D$ is a noise coefficient, then one obtains the SDE

$$
\begin{aligned}
d \mathbf{x}=\left(\begin{array}{c}
d x \\
d y \\
d \theta
\end{array}\right) & =\left(\begin{array}{c}
\frac{r}{2}\left(\omega_{1}+\omega_{2}\right) \cos \theta \\
\frac{r}{2}\left(\omega_{1}+\omega_{2}\right) \sin \theta \\
\frac{r}{\ell}\left(\omega_{1}-\omega_{2}\right)
\end{array}\right) d t \\
& +\sqrt{D}\left(\begin{array}{cc}
\frac{r}{2} \cos \theta & \frac{r}{2} \cos \theta \\
\frac{r}{2} \sin \theta & \frac{r}{2} \sin \theta \\
\frac{r}{\ell} & -\frac{r}{\ell}
\end{array}\right)\left(\begin{array}{l}
d w_{1} \\
d w_{2}
\end{array}\right) .
\end{aligned}
$$

In this paper, we focus on the distributions for the robot, which deterministically would be driving straight or driving along an arc of constant curvature. For deterministic driving straight forward at speed $v$, the constant wheel speeds are

$$
\omega_{1}=\omega_{2}=\frac{v}{r} .
$$

For deterministic driving along an arc of radius $a$ counterclockwise at rate $\dot{\alpha}$, the wheel speeds can be shown to be

$$
\omega_{1}=\frac{\dot{\alpha}}{r}\left(a+\frac{\ell}{2}\right), \quad \omega_{2}=\frac{\dot{\alpha}}{r}\left(a-\frac{\ell}{2}\right) .
$$

\section{REVIEW OF RIGID-BODY Motions}

The planar special Euclidean group, $\mathrm{G}=S E(2)$, is the semidirect product of the plane, $\mathbb{R}^{2}$, with the special orthogonal group, $S O(2)$. The elements of $S E(2)$ can be represented with a rotational part $R \in S O(2)$ and a translational part $\mathbf{t}=\left[t_{1}, t_{2}\right]^{T}$ as $3 \times 3$ homogeneous transformation matrices

$$
g=\left(\begin{array}{cc}
R & \mathbf{t} \\
\mathbf{0}^{T} & 1
\end{array}\right) \in S E(2),
$$

where the group operator $\circ$ is matrix multiplication.

We will also make use of the Lie algebra $s e(2)$ associated with $S E(2)$. For a vector $\mathbf{x}=\left[v_{1}, v_{2}, \alpha\right]^{T}$, an element $X$ of the Lie algebra $s e(2)$ can then be expressed as

$$
X=\hat{\mathbf{x}}=\left(\begin{array}{ccc}
0 & -\alpha & v_{1} \\
\alpha & 0 & v_{2} \\
0 & 0 & 0
\end{array}\right) \quad \text { and } \quad X^{\vee}=\mathbf{x}
$$

where the $\wedge$ and $\vee$ operators allow us to map from $R^{3}$ to $s e(2)$ and back.

By using the matrix exponential $\exp (\cdot)$ on elements of $s e(2)$, we can obtain group elements of $S E(2)$ as

$$
\begin{aligned}
g\left(v_{1}, v_{2}, \alpha\right) & =\exp (X) \\
& =\left(\begin{array}{ccc}
\cos \alpha & -\sin \alpha & t_{1} \\
\sin \alpha & \cos \alpha & t_{2} \\
0 & 0 & 1
\end{array}\right),
\end{aligned}
$$

where

$$
\begin{aligned}
& t_{1}=\left[v_{2}(-1+\cos \alpha)+v_{1} \sin \alpha\right] / \alpha \quad \text { and } \\
& t_{2}=\left[v_{1}(1-\cos \alpha)+v_{2} \sin \alpha\right] / \alpha .
\end{aligned}
$$

Since we are using the exponential in this formulation, we will refer to the coordinates $\left(v_{1}, v_{2}, \alpha\right)$ as exponential coordinates. 
We can obtain the vector of exponential coordinates $\mathbf{x}$ from the group element $g \in S E(2)$ from

$$
\mathbf{x}=(\log (g))^{\vee},
$$

where $\log (\cdot)$ is the matrix logarithm.

Given a time-dependent rigid body motion $g(t)$, the spatial velocity as seen in the body-fixed frame is given as the quantity

$$
g^{-1} \dot{g}=\left(\begin{array}{cc}
R^{T} \dot{R} & R^{T} \dot{\mathbf{t}} \\
\mathbf{0}^{T} & 0
\end{array}\right) \in \operatorname{se}(2),
$$

where the dot represents a time derivative.

We define the adjoint operator $A d(g)$ to satisfy [7]

$$
A d(g) \mathbf{x}=\log ^{\vee}\left(g \circ \exp (X) \circ g^{-1}\right) .
$$

For $S E(2)$, the adjoint matrix is given by

$$
A d(g)=\left(\begin{array}{cc}
R & M \mathbf{t} \\
\mathbf{0}^{T} & 1
\end{array}\right) \text { where } \quad M=\left(\begin{array}{cc}
0 & 1 \\
-1 & 0
\end{array}\right) .
$$

We define another adjoint operator $a d(X)$ to satisfy [7]

$$
\operatorname{ad}(X) \mathbf{y}=([X, Y])^{\vee},
$$

where $[X, Y]=X Y-Y X$ is the Lie bracket. This adjoint matrix for $s e(2)$ is given by

$$
\operatorname{ad}(X)=\left(\begin{array}{cc}
-\alpha M & M \mathbf{v} \\
\mathbf{0}^{T} & 0
\end{array}\right)
$$

where $\mathbf{v}=\left[v_{1}, v_{2}\right]^{T}$. The two adjoint matrices are related by

$$
\operatorname{Ad}(\exp (X))=\exp (\operatorname{ad}(X)) .
$$

The Baker-Campbell-Hausdorff (BCH) formula [9] is a useful expression that relates the matrix exponential with the Lie bracket. The $\mathrm{BCH}$ is given by

$$
\begin{aligned}
Z(X, Y) & =\log (\exp (X) \circ \exp (Y)) \\
& =X+Y+\frac{1}{2}[X, Y]+\ldots
\end{aligned}
$$

If the $\vee$ operator is applied to this formula, we obtain

$$
\mathbf{z}=\mathbf{x}+\mathbf{y}+\frac{1}{2} a d(X) \mathbf{y}+\ldots
$$

\section{Means, Covariances and Gaussian DISTRIBUTIONS}

For a vector $\mathbf{x} \in \mathbb{R}^{n}$, the mean $\tilde{\boldsymbol{\mu}}$ and covariance $\tilde{\Sigma}$ about the mean for the pdf $f(\mathbf{x})$ are given respectively as 1

$$
\begin{aligned}
0 & =\int_{\mathbb{R}^{n}}(\mathbf{x}-\tilde{\boldsymbol{\mu}}) f(\mathbf{x}) d \mathbf{x} \\
\tilde{\Sigma} & =\int_{\mathbb{R}^{n}}(\mathbf{x}-\tilde{\boldsymbol{\mu}})(\mathbf{x}-\tilde{\boldsymbol{\mu}})^{T} f(\mathbf{x}) d \mathbf{x}
\end{aligned}
$$

A multidimensional Gaussian probability density function with mean $\tilde{\boldsymbol{\mu}}$ and covariance matrix $\tilde{\Sigma}$ is defined as

$$
f(\mathbf{x} ; \tilde{\boldsymbol{\mu}}, \tilde{\Sigma})=\frac{1}{\tilde{c}(\tilde{\Sigma})} \exp \left[-\frac{1}{2}(\mathbf{x}-\tilde{\boldsymbol{\mu}})^{T} \tilde{\Sigma}^{-1}(\mathbf{x}-\tilde{\boldsymbol{\mu}})\right],
$$

${ }^{1}$ We will use a 'tilde' ( ) to represent quantities associated with the Gaussian in Cartesian coordinates. where $\exp (\cdot)$ is the usual scalar exponential function and $\tilde{c}(\tilde{\Sigma})$ is a normalizing factor to ensure $f(\mathbf{x} ; \tilde{\boldsymbol{\mu}}, \tilde{\Sigma})$ is a pdf. In $[19]$,

$$
\tilde{c}(\tilde{\Sigma})=(2 \pi)^{n / 2}|\operatorname{det} \tilde{\Sigma}|^{1 / 2} .
$$

These definitions can be naturally extended to matrix Lie groups as in [25]. Given a group $G$ with operation $\circ$, the mean $\mu \in G$ of a pdf $f(g)$ is defined to satisfy

$$
\int_{G} \log ^{\vee}\left(\mu^{-1} \circ g\right) f(g) d g=\mathbf{0} .
$$

The covariance about the mean is defined as

$$
\Sigma=\int_{G} \log ^{\vee}\left(\mu^{-1} \circ g\right)\left[\log ^{\vee}\left(\mu^{-1} \circ g\right)\right]^{T} f(g) d g .
$$

A multidimensional Gaussian for Lie groups can be defined as

$$
f(g ; \mu, \Sigma)=\frac{1}{c(\Sigma)} \exp \left[-\frac{1}{2} \mathbf{y}^{T} \Sigma^{-1} \mathbf{y}\right],
$$

where $\mathbf{y}=\log \left(\mu^{-1} \circ g\right)^{\vee}$ and $c(\Sigma)$ is a normalizing factor. For highly concentrated distributions (i.e. the distribution decays rapidly as you move away from the mean $), c(\Sigma) \approx \tilde{c}(\tilde{\Sigma})$.

\section{GAUSSIAN COMPARISONS}

In this section, we compare the Gaussian in Cartesian coordinates defined in (19) to the one defined in 23) in exponential coordinates for the differential-drive car moving along a straight path and moving along an arc. For all the examples in the remainder of this paper, the wheel base $\ell$ is 0.200 and the radius $r$ of each wheel is 0.033 . To obtain a set of sample frames representing the true distribution of this system, we numerically integrated the stochastic differential equation given in (2) 10,000 times using a modified version of the Euler-Maruyama method [12] with a time step of $d t=0.001$ for a total time of $T=1$ second.

Using these sample data points, we can approximate the mean and covariance for Cartesian coordinates with

$$
\begin{aligned}
\tilde{\boldsymbol{\mu}} & =\frac{1}{N} \sum_{i=1}^{N} \mathbf{x}_{i}, \quad \text { and } \\
\tilde{\Sigma} & =\frac{1}{N} \sum_{i=1}^{N}\left(\mathbf{x}_{i}-\tilde{\boldsymbol{\mu}}\right)\left(\mathbf{x}_{i}-\tilde{\boldsymbol{\mu}}\right)^{T},
\end{aligned}
$$

where $\mathbf{x}_{i}=\left[x_{i}, y_{i}, \theta_{i}\right]^{T}$ and $N$ is the number of sample points.

The mean for exponential coordinates defined in (21) can be approximated with the recursive formula,

$$
\mu=\mu \circ \exp \left[\frac{1}{N} \sum_{i=1}^{N} \log \left(\mu^{-1} \circ g_{i}\right)\right] .
$$

The covariance matrix in exponential coordinates defined in (22) can be estimated with

$$
\Sigma=\frac{1}{N} \sum_{i=1}^{N} \mathbf{y}_{i} \mathbf{y}_{i}^{T},
$$

where $\mathbf{y}_{i}=\left[\log \left(\mu^{-1} \circ g_{i}\right)\right]^{\vee}$. 

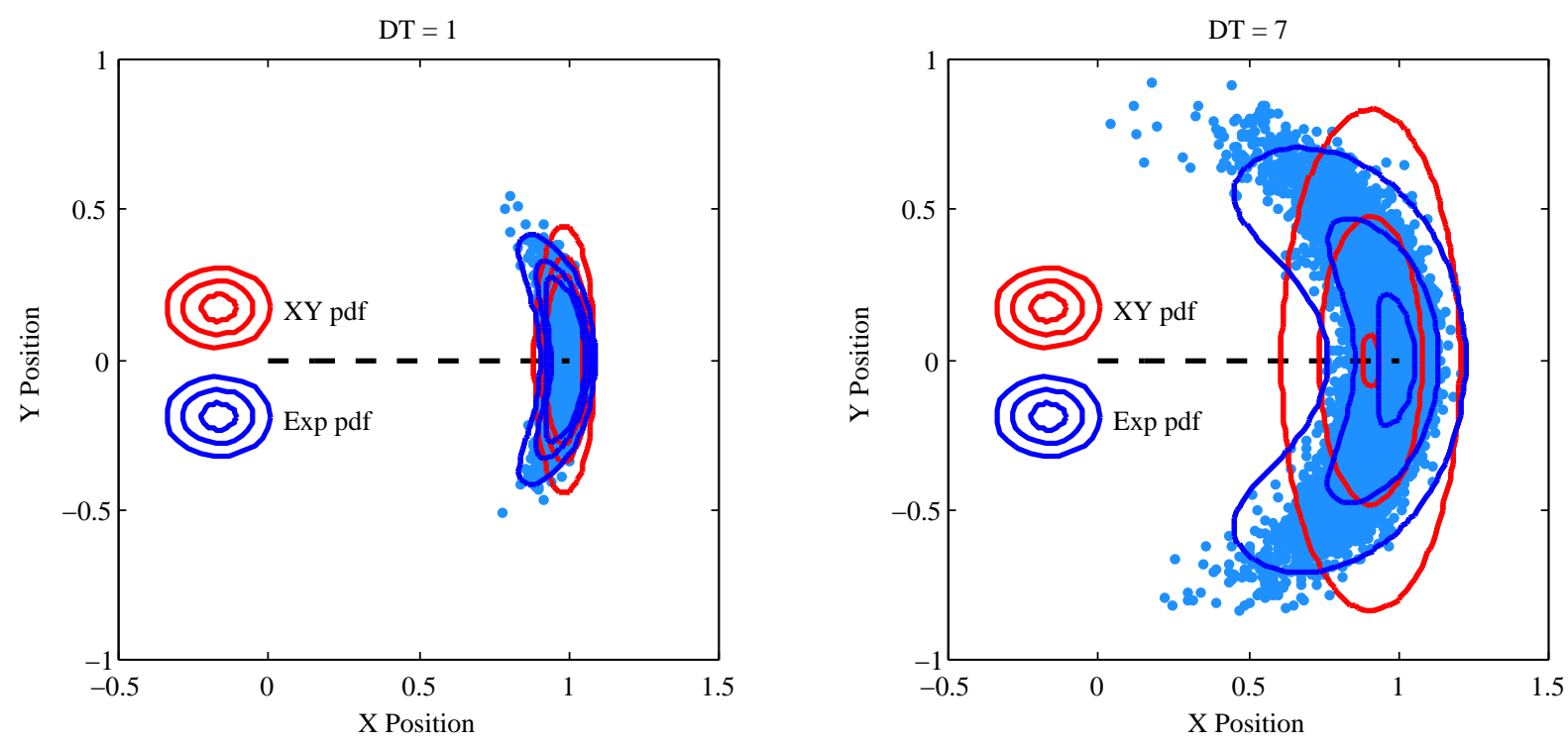

Fig. 2. Distributions for the differential drive robot moving ideally along a straight line with diffusion constant $D T=1$ (left) and $D T=7$ (right). Both plots have pdf contours of Gaussians of best fit in exponential coordinates and Cartesian coordinates marginalized over the heading.
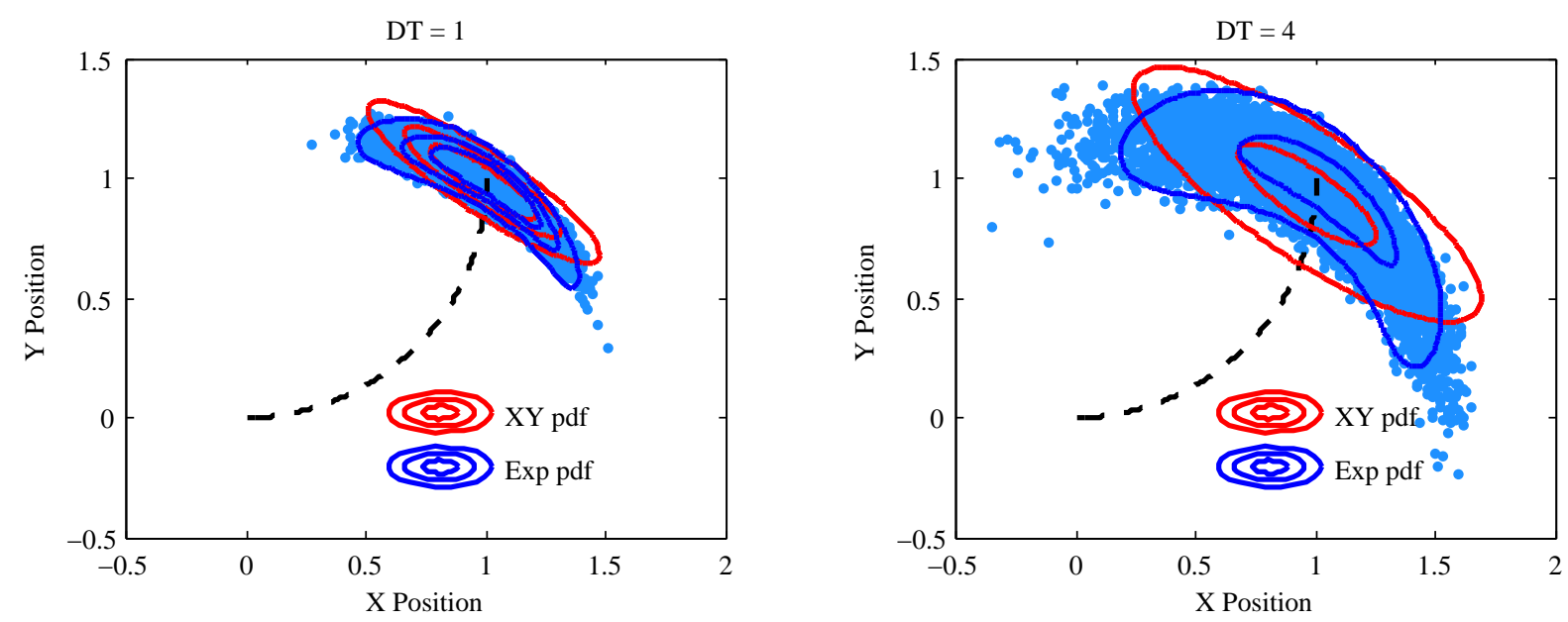

Fig. 3. Distributions for the differential-drive robot moving ideally along a constant-curvature arc with diffusion constant $D T=1$ (left) and $D T=4$ (right). Both plots have pdf contours of Gaussians of best fit in exponential coordinates and Cartesian coordinates marginalized over the heading.

The sample mean and covariance for each method parameterizes the associated pdf. These pdfs were marginalized over orientation and contours of equal pdf values were plotted along with the sampled end positions of the simulations. Fig. 2 and Fig. 3 present these contours for various values of $D T$ for the driving straight and driving along an arc examples, where $D$ is a diffusion coefficient and $T$ is the total drive time. Here $T$ is equal to one. It is evident that the Gaussian in exponential coordinates fits the sampled data more accurately for both cases, especially as uncertainty increases. If we take the poses of the sampled data, represent them in exponential coordinates, and look at the $\left(v_{1}, v_{2}\right)$ values as shown in Fig. 4, it is clear that the result is approximately elliptical. To numerically verify that the Gaussian in exponential coordinates is a better fit for the sample data than the Gaussian in
Cartesian coordinates, we performed a log-likelihood ratio test for various values of the diffusion coefficient $D$ as shown in Fig. 5. Note that for small diffusion values, the log-likelihood ratio is close to one indicating that both Gaussian models perform approximately the same. However, as the uncertainty is increased the Gaussian in (23) in exponential coordinates performs better than the Gaussian in Cartesian coordinates.

\section{Propagation with Exponential Coordinates}

In this section, we describe how to propagate the mean and covariance defined in (21) and (22) with closed-form estimation formulas. By using the SDE (2), we can derive 
another stochastic differential equation as

$$
\begin{aligned}
& \left(g^{-1} \dot{g}\right)^{\vee} d t=\left(\begin{array}{c}
\cos (\alpha) d x+\sin (\alpha) d y \\
-\sin (\alpha) d x+\cos (\alpha) d y \\
d \alpha
\end{array}\right) \\
& =\left(\begin{array}{c}
\frac{r}{2}\left(\omega_{1}+\omega_{2}\right) \\
0 \\
\frac{r}{\ell}\left(\omega_{1}-\omega_{2}\right)
\end{array}\right) d t+\sqrt{D}\left(\begin{array}{cc}
\frac{r}{2} & \frac{r}{2} \\
0 & 0 \\
\frac{r}{\ell} & -\frac{r}{\ell}
\end{array}\right)\left(\begin{array}{c}
d w_{1} \\
d w_{2}
\end{array}\right) .
\end{aligned}
$$

This will be written in short-hand as

$$
\left(g^{-1} \dot{g}\right)^{\vee} d t=\mathbf{h} d t+H d \mathbf{w} .
$$

When rigid-body transformations are close to the identity, the $S E(2)$-mean and $S E(2)$-covariance defined in 21 and (22) can be approximated with [19]

$$
\begin{gathered}
\mu(t)=\exp \left(\int_{0}^{t} \hat{\mathbf{h}} d \tau\right), \quad \text { and } \\
\Sigma(t)=\int_{0}^{t} A d\left(\mu^{-1}(\tau)\right) H H^{T} A d^{T}\left(\mu^{-1}(\tau)\right) d \tau
\end{gathered}
$$

where $H H^{T}$ is a constant diffusion matrix.

For the straight driving example $\left(\omega_{1}=\omega_{2}=\omega\right)$, we have

$$
\mu(t)=\left(\begin{array}{ccc}
1 & 0 & r \omega t \\
0 & 1 & 0 \\
0 & 0 & 1
\end{array}\right)
$$

We can solve the integral in (31) in closed form as

$$
\Sigma(t)=\left(\begin{array}{ccc}
\frac{1}{2} D r^{2} t & 0 & 0 \\
0 & \frac{2 D \omega^{2} r^{4} t^{3}}{3 \ell^{2}} & \frac{D \omega r^{3} t^{2}}{\ell^{2}} \\
0 & \frac{D \omega r^{3} t^{2}}{\ell^{2}} & \frac{2 D r^{2} t}{\ell^{2}}
\end{array}\right) .
$$

For the constant curvature case $\left(\omega_{1} \neq \omega_{2}\right)$ with (4), we have

$$
\mu(t)=\left(\begin{array}{ccc}
\cos (\dot{\alpha} t) & -\sin (\dot{\alpha} t) & a \sin (\dot{\alpha} t) \\
\sin (\dot{\alpha} t) & \cos (\dot{\alpha} t) & a(1-\cos (\dot{\alpha} t)) \\
0 & 0 & 1
\end{array}\right) .
$$

The closed-form $S E(2)$-covariance matrix is then

$$
\begin{aligned}
& \Sigma(t)=\left(\begin{array}{lll}
\sigma_{11} & \sigma_{12} & \sigma_{13} \\
\sigma_{21} & \sigma_{22} & \sigma_{23} \\
\sigma_{31} & \sigma_{32} & \sigma_{33}
\end{array}\right), \text { where } \\
\sigma_{11}= & \frac{c}{8}\left[\left(4 a^{2}+\ell^{2}\right)(2 \dot{\alpha} t+\sin (2 \dot{\alpha} t))\right. \\
& \left.+16 a^{2}(\dot{\alpha} t-2 \sin (\dot{\alpha} t))\right], \\
\sigma_{12}= & \sigma_{21}=\frac{-c}{2}\left[4 a^{2}(-1+\cos (\dot{\alpha} t))+\ell^{2}\right] \sin \left(\frac{\dot{\alpha} t}{2}\right)^{2}, \\
\sigma_{13}= & \sigma_{31}=2 c a(\dot{\alpha} T-\sin (\dot{\alpha} t)), \\
\sigma_{22}= & -\frac{c}{8}\left(4 a^{2}+\ell^{2}\right)(-2 \dot{\alpha} t+\sin (2 \dot{\alpha} t)), \\
\sigma_{23}= & \sigma_{32}=-2 c a(-1+\cos (\dot{\alpha} t)), \\
\sigma_{33}= & 2 c \dot{\alpha}, \quad \text { and } \\
c= & \frac{D r^{2}}{\ell^{2} \dot{\alpha}} .
\end{aligned}
$$
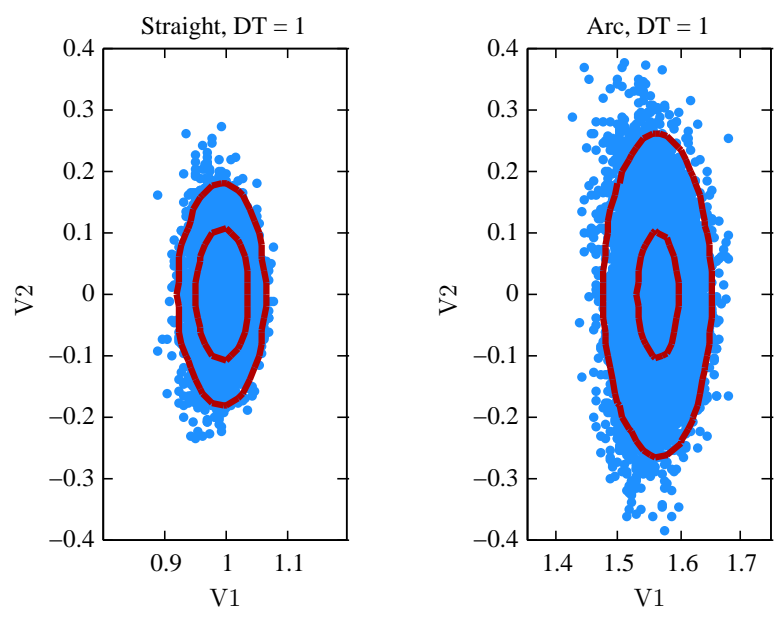

Fig. 4. Distributions as a function of exponential coordinates $\left(v_{1}, v_{2}\right)$ with pdf contours marginalized over the heading for the Gaussian in exponential coordinates for driving straight (left) and driving along an arc (right) with diffusion $D T=1$.

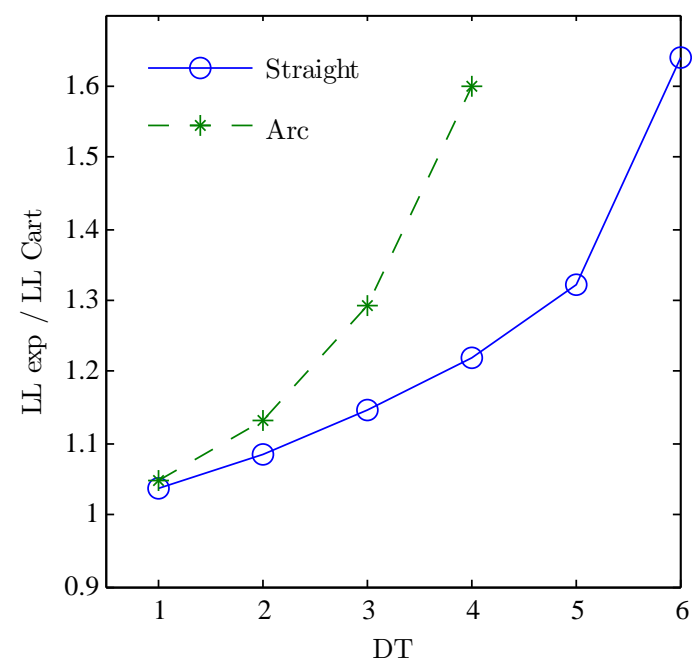

Fig. 5. Log-likelihood ratio test of the Gaussian in exponential coordinates to the Gaussian in Cartesian coordinates as a function of the diffusion $D T$.

To demonstrate the accuracy of this propagation method, we calculated the $S E(2)$-mean and $S E(2)$-covariance from 10,000 sample data frames using (26) and (27) and from the propagation formulas. For the straight driving example with $r \omega=1$ and $D T=1$, the mean and covariance of the data are

$$
\begin{aligned}
\mu_{\text {data }} & =\left(\begin{array}{ccc}
1.0000 & 0.0003 & 1.0000 \\
-0.0003 & 1.0000 & 0.0000 \\
0 & 0 & 1
\end{array}\right) \text { and } \\
\Sigma_{\text {data }} & =\left(\begin{array}{ccc}
0.0006 & 0.0000 & -0.0001 \\
0.0000 & 0.0184 & 0.0276 \\
-0.0001 & 0.0276 & 0.0551
\end{array}\right) .
\end{aligned}
$$

The propagated mean and covariance for these parameters are 


$$
\begin{gathered}
\mu_{\text {prop }}=\left(\begin{array}{lll}
1 & 0 & 1 \\
0 & 1 & 0 \\
0 & 0 & 1
\end{array}\right) \text { and } \\
\Sigma_{\text {prop }}=\left(\begin{array}{ccc}
0.0006 & 0 & 0 \\
0 & 0.0184 & 0.0276 \\
0 & 0.0276 & 0.0553
\end{array}\right) .
\end{gathered}
$$

If we increase the noise to $D T=7$, the mean and covariance from sample data are

$$
\begin{gathered}
\mu_{\text {data }}=\left(\begin{array}{ccc}
1.0000 & 0.0011 & 1.0009 \\
-0.0011 & 1.0000 & -0.0002 \\
0 & 0 & 1
\end{array}\right) \text { and } \\
\Sigma_{\text {data }}=\left(\begin{array}{lll}
0.0068 & 0.0000 & 0.0002 \\
0.0000 & 0.1278 & 0.1943 \\
0.0002 & 0.1943 & 0.3883
\end{array}\right) .
\end{gathered}
$$

The propagated mean and covariance are

$$
\begin{gathered}
\mu_{\text {prop }}=\left(\begin{array}{lll}
1 & 0 & 1 \\
0 & 1 & 0 \\
0 & 0 & 1
\end{array}\right) \text { and } \\
\Sigma_{\text {prop }}=\left(\begin{array}{ccc}
0.0039 & 0 & 0 \\
0 & 0.1290 & 0.1935 \\
0 & 0.1935 & 0.3869
\end{array}\right) .
\end{gathered}
$$

Similar accuracy can be seen for the constant curvature case.

\section{Fusion With EXPonential COORDINATES}

Here we consider the fusion of banana distributions for $N$ robots moving in formation. To begin, consider only two such robots, $i$ and $j$. The actual (unknown) pose of these robots at time $t$ are $g_{i}$ and $g_{j}$. At time $t=0$, the known initial poses are $a_{i}$ and $a_{j}$, and the banana distributions for each robot diffuse so as to result in distributions $f\left(a_{i}^{-1} \circ g_{i} ; \mu_{i}, \Sigma_{i}\right)$ and $f\left(a_{j}^{-1} \circ g_{j} ; \mu_{j}, \Sigma_{j}\right)$. The means and covariances of these two distributions can be estimated using the propagation formulas from the previous section.

Let us assume that an exact relative measurement between $i$ and $j$ is taken so that

$$
m_{i j}=g_{i}^{-1} \circ g_{j}
$$

is known. Then the distribution of $g_{i}$ becomes with a form of Bayesian fusion

$$
p_{i j}\left(g_{i}\right)=f\left(a_{i}^{-1} \circ g_{i} ; \mu_{i}, \Sigma_{i}\right) \cdot f\left(a_{j}^{-1} \circ g_{i} \circ m_{i j} ; \mu_{j}, \Sigma_{j}\right) .
$$

Noting that $m_{j i}=m_{i j}^{-1}$ and switching the roles of $i$ and $j$ gives the same expression for $p_{j i}\left(g_{j}\right)$. Our goal in this fusion problem is to write this new fused distribution in the form

$$
p_{i j}\left(g_{i}\right)=f\left(a_{i}^{-1} \circ g_{i} ; \mu_{i j}, \Sigma_{i j}\right),
$$

and more generally, $p_{1,2, \ldots, N}\left(g_{i}\right)$. To save space in this section, we will occasionally remove the $\circ$ when the operation is clear.

We can rewrite $p_{i j}\left(g_{i}\right)$ in (45) as

$p_{i j}\left(g_{i}\right)=f\left(\mu_{i}^{-1} a_{i}^{-1} g_{i} ; I, \Sigma_{i}\right) \cdot f\left(\mu_{j}^{-1} a_{j}^{-1} g_{i} m_{i j} ; I, \Sigma_{j}\right)$.
Making the substitution $h=\mu_{i}^{-1} \circ a_{i}^{-1} \circ g_{i}$, we have

$$
p_{i j}\left(a_{i} \mu_{i} h\right)=f\left(h ; I, \Sigma_{i}\right) \cdot f\left(m_{i j}^{-1} q h m_{i j} ; I, \Sigma_{j}\right),
$$

where $q=m_{i j} \circ \mu_{j}^{-1} \circ a_{j}^{-1} \circ a_{i} \circ \mu_{i}$. We rewrite [46] as

$$
p_{i j}\left(a_{i} \mu_{i} h\right)=f\left(\mu_{i} h ; \mu_{i j}, \Sigma_{i j}\right)=f\left(h ; \mu_{i j}^{\prime}, \Sigma_{i j}\right),
$$

where $\mu_{i j}^{\prime}=\mu_{i}^{-1} \circ \mu_{i j}$. Our goal is to find closed-form expressions for $\mu_{i j}^{\prime}$ and $\Sigma_{i j}$.

The exponents of all three Gaussian distributions $(f)$ in 48 and (49) are all scalars and thus we have, to within a constant $C$,

$$
\begin{aligned}
& {\left[\log ^{\vee}\left(e^{X_{i j}} e^{Y}\right)\right]^{T} \Sigma_{i j}^{-1}\left[\log ^{\vee}\left(e^{X_{i j}} e^{Y}\right)\right]} \\
& =C+\left[\log ^{\vee}\left(e^{X_{i}} e^{Y}\right)\right]^{T} \Sigma_{i}^{-1}\left[\log ^{\vee}\left(e^{X_{i}} e^{Y}\right)\right] \\
& +\left[\log ^{\vee}\left(m_{i j}^{-1} e^{X_{j}} e^{Y} m_{i j}\right)\right]^{T} \Sigma_{j}^{-1}\left[\log ^{\vee}\left(m_{i j}^{-1} e^{X_{j}} e^{Y} m_{i j}\right)\right],
\end{aligned}
$$

where $e^{X_{i j}}=\exp \left(X_{i j}\right)=\left(\mu_{i j}^{\prime}\right)^{-1}, e^{X_{i}}=\exp \left(X_{i}\right)=I$, $e^{X_{j}}=\exp \left(X_{j}\right)=q$ and $e^{Y}=\exp (Y)=h$. Note that

$$
\log ^{\vee}\left(m_{i j}^{-1} e^{X_{j}} e^{Y} m_{i j}\right)=A d\left(m_{i j}^{-1}\right) \log ^{\vee}\left(e^{X_{j}} e^{Y}\right) .
$$

We assume that $\mu_{i j}^{\prime}, q$ and $h$ are small such that the $\mathrm{BCH}$ expansion can be approximated with only the first three terms

$$
\log ^{\vee}(\exp (X) \exp (Y)) \approx \mathbf{x}+\mathbf{y}+\frac{1}{2} a d(X) \mathbf{y} .
$$

Since $\operatorname{ad}(X) \mathbf{x}=0$, we can rewrite (52) as

$$
\log ^{\vee}(\exp (X) \exp (Y))=\left(I+\frac{1}{2} a d(X)\right)(\mathbf{x}+\mathbf{y}) .
$$

Since we renormalize, we rewrite (50) ignoring the constant $C$ as

$$
\begin{aligned}
& \left(\mathbf{x}_{i j}+\mathbf{y}\right)^{T} \Gamma_{i j}^{T} \Sigma_{i j}^{-1} \Gamma_{i j}\left(\mathbf{x}_{i j}+\mathbf{y}\right) \\
& =\left(\mathbf{x}_{i}+\mathbf{y}\right)^{T} \Gamma_{i}^{T} \Sigma_{i}^{-1} \Gamma_{i}\left(\mathbf{x}_{i}+\mathbf{y}\right) \\
& +\left(\mathbf{x}_{j}+\mathbf{y}\right)^{T} \Gamma_{j}^{T} A d^{-T}\left(m_{i j}\right) \Sigma_{j}^{-1} A d^{-1}\left(m_{i j}\right) \Gamma_{j}\left(\mathbf{x}_{j}+\mathbf{y}\right)
\end{aligned}
$$

where $\Gamma_{k}=I+\frac{1}{2} a d\left(X_{k}\right)$. Let

$$
\begin{aligned}
S_{i j} & =\Gamma_{i j}^{T} \Sigma_{i j}^{-1} \Gamma_{i j}, \quad S_{i}=\Gamma_{i}^{T} \Sigma_{i}^{-1} \Gamma_{i}, \quad \text { and } \\
S_{j} & =\Gamma_{j}^{T} A d^{-T}\left(m_{i j}\right) \Sigma_{j}^{-1} A d^{-1}\left(m_{i j}\right) \Gamma_{j} .
\end{aligned}
$$

We can then combine like terms and solve for the following closed-form formulas

$$
\begin{aligned}
S_{i j} & =S_{i}+S_{j}, \\
\mathbf{x}_{i j} & =S_{i j}^{-1}\left(S_{i} \mathbf{x}_{i}+S_{j} \mathbf{x}_{j}\right), \quad \text { and } \\
\Sigma_{i j} & =\Gamma_{i j} S_{i j}^{-1} \Gamma_{i j}^{T}
\end{aligned}
$$

which have a similar form to the product of Gaussians on $\mathbb{R}^{n}$. We can then extract the desired mean $\mu_{i j}$ as

$$
\mu_{i j}=\mu_{i} \circ \exp \left(-\hat{\mathbf{x}}_{i j}\right) .
$$

These equations can be used recursively for multiple robot systems with multiple measurements. 
These formulas can be contrasted with those used traditionally for Bayesian fusion of multivariate Gaussians on $\mathbb{R}^{3}$,

$$
\begin{aligned}
& f\left(\mathbf{x}_{i} ; \tilde{\mathbf{a}}_{i}+\tilde{\boldsymbol{\mu}}_{i j}, \tilde{\Sigma}_{i j}\right)= \\
& \quad f\left(\mathbf{x}_{i} ; \tilde{\mathbf{a}}_{i}+\tilde{\boldsymbol{\mu}}_{i}, \tilde{\Sigma}_{i}\right) \cdot f\left(\mathbf{x}_{i} ; \tilde{\mathbf{a}}_{j}+\tilde{\boldsymbol{\mu}}_{j}-\tilde{\mathbf{m}}_{i j}, \tilde{\Sigma}_{j}\right)
\end{aligned}
$$

where $\tilde{\mathbf{m}}_{i j}=\mathbf{x}_{j}-\mathbf{x}_{i}$,

$\tilde{\Sigma}_{i j}^{-1}=\tilde{\Sigma}_{i}^{-1}+\tilde{\Sigma}_{j}^{-1}, \quad$ and

$\tilde{\boldsymbol{\mu}}_{i j}=\tilde{\Sigma}_{i j}\left[\tilde{\Sigma}_{i}^{-1}\left(\tilde{\mathbf{a}}_{i}+\tilde{\boldsymbol{\mu}}_{i}\right)+\tilde{\Sigma}_{j}^{-1}\left(\tilde{\mathbf{a}}_{j}+\tilde{\boldsymbol{\mu}}_{j}-\tilde{\mathbf{m}}_{i j}\right)\right]-\tilde{\mathbf{a}}_{i}$.

The propagation and fusion formulas were tested on a three robot system driving in a triangular formation with diffusion constant $D T=3$ as shown in Fig. 6 Sample data from integrating the SDE in (2) many times are represented by blue dots similar to Fig. 1(a). We integrated the SDE in (2) once from each starting position to represent a set of three true poses represented by green circles. From these true poses, we generated the exact measurements between each robot. The pdf contours from the fused Gaussians in exponential coordinates marginalized over the heading are also shown in the figure. Note that the fused pdf contours are not trying to match the banana distributions, but instead give a better estimate of the location of the robots based on the exact measurements.

These results obtained using propagation and fusion on Gaussians on exponential coordinates were compared with the fusion of Gaussians on Cartesian coordinates. For comparison, we let $x_{i}^{*}$ be the true pose $\left(x_{i}, y_{i}, \theta_{i}\right)^{T}$ in Cartesian coordinates for the $i$ th robot. Then, $x_{i}^{e}$ and $x_{i}^{c}$ are the poses in Cartesian coordinates associated with the means from the fused Gaussians in exponential and Cartesian coordinates, respectively. For Fig. 6, these poses are given as:

$$
\begin{aligned}
& \mathbf{x}_{1}^{*}=\left(\begin{array}{c}
2.043 \\
0.090 \\
-0.034
\end{array}\right), \mathbf{x}_{1}^{\mathrm{e}}=\left(\begin{array}{c}
2.010 \\
0.039 \\
-0.054
\end{array}\right), \mathbf{x}_{1}^{\mathrm{c}}=\left(\begin{array}{c}
1.976 \\
0.146 \\
0.132
\end{array}\right) \\
& \mathbf{x}_{2}^{*}=\left(\begin{array}{c}
1.001 \\
0.840 \\
-0.128
\end{array}\right), \mathbf{x}_{2}^{\mathrm{e}}=\left(\begin{array}{c}
0.993 \\
0.836 \\
-0.182
\end{array}\right), \mathbf{x}_{2}^{\mathrm{c}}=\left(\begin{array}{c}
0.934 \\
0.896 \\
0.037
\end{array}\right) \\
& \mathbf{x}_{3}^{*}=\left(\begin{array}{c}
1.033 \\
-1.077 \\
-0.283
\end{array}\right), \mathbf{x}_{3}^{\mathrm{e}}=\left(\begin{array}{c}
0.977 \\
-1.106 \\
-0.303
\end{array}\right), \mathbf{x}_{3}^{\mathrm{c}}=\left(\begin{array}{c}
0.966 \\
-1.021 \\
-0.117
\end{array}\right) .
\end{aligned}
$$

It is clear that the means for the Gaussian model in exponential coordinates is closer to the true values. Moreover, if we examine the covariances associated with both models, it is apparent that the covariances of the exponential model have smaller magnitude than those for the Cartesian Gaussian. For example, the covariances of the fused distributions for the first robot are

$$
\Sigma_{1}=\left(\begin{array}{ccc}
0.0006 & -0.0000 & -0.0001 \\
-0.0000 & 0.0060 & 0.0011 \\
-0.0001 & 0.0011 & 0.0008 \\
0.0013 & -0.0002 & -0.0003 \\
-0.0002 & 0.0166 & 0.0267 \\
-0.0003 & 0.0267 & 0.0571
\end{array}\right) \text { and }
$$

Thus, the fused exponential coordinate Gaussians' results are more highly focused than the Cartesian ones.

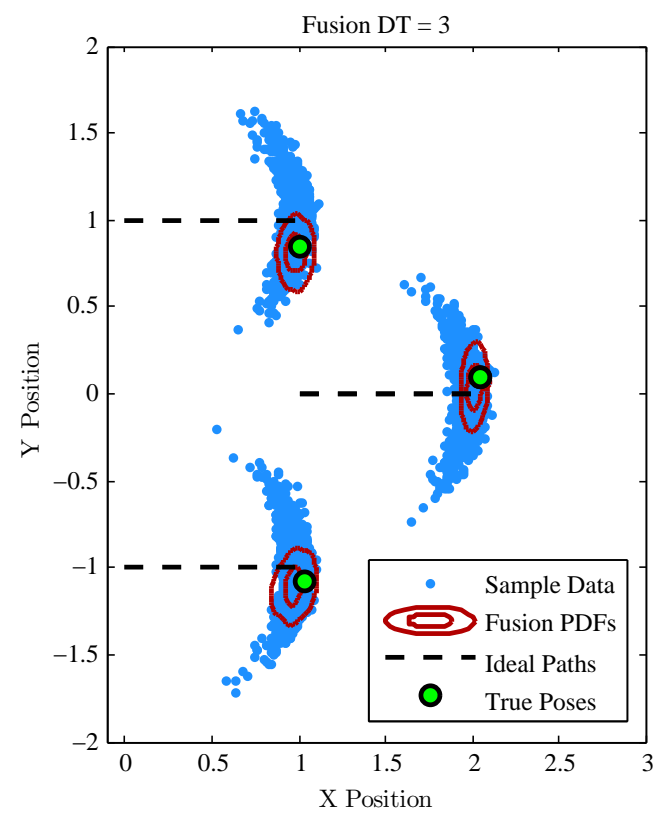

Fig. 6. Fusion of Gaussian in exponential coordinates for three robots driving in formation with true positions marked with a filled in circle.

\section{CONCLUSION}

A comparison between the commonly used multivariate Gaussian model on $(x, y, \theta)$ and one based on the coordinates of the Lie algebra (or exponential coordinates) has been provided. These models were examined in the context of the stochastic kinematic model of a differential-drive robot. Through numerical examples, we demonstrated that the model based on exponential coordinates is able to capture the underlying distribution more accurately and more precisely. This held true for trajectories that were both straight and circular. In addition, a closed-form approximation was given for propagating the mean and covariance for paths consisting of straight segments and arcs of constant curvature. This allows the mean and covariance to be estimated quickly.

Finally, we presented a closed-form approximation for multiplying two Gaussian pdfs in exponential coordinates. Multiplying two pdfs allows us to effectively fuse information obtained from two different sources. In the case of the example provided, these sources were forward propagation of the stochastic kinematic model and relative measurements between robots. The pdfs obtained using this method provided significantly increased accuracy when compared with those from just forward propagation.

The methods and models described above can be used to improve current state of the art SLAM algorithms. We hope to use these methods to develop a new filter for $\mathrm{SE}(2)$ similar to the Kalman filter. Also, we would like to develop new approaches for fusing data from different models together. For example, if a Gaussian on exponential coordinates is used to model forward propagation, we want to fuse it with noisy measurements from a Gaussian on Cartesian coordinates. 


\section{ACKNOWLEDGMENTS}

This work was funded in part by NSF grant IIS-0915542 "RI: Small: Robotic Inspection, Diagnosis, and Repair" and Andrew Long's NDSEG fellowship.

\section{REFERENCES}

[1] M. Agrawal. A Lie algebraic approach for consistent pose registration for general euclidean motion. In Proc. of IEEE Int'l Conf. on Intelligent Robots and Systems (IROS), pages 1891-1897, 2006.

[2] T. Bailey and H. Durrant-Whyte. Simultaneous localization and mapping (SLAM): Part II. IEEE Robotics and Automation Magazine, 13(3):108-117, 2006.

[3] T. Bailey, J. Nieto, J. Guivant, M. Stevens, and E. Nebot. Consistency of the EKF-SLAM algorithm. In Proc. of IEEE Int'l Conf. on Intelligent Robots and Systems (IROS), pages 3562-3568, 2006.

[4] G. Baldwin, R. Mahony, J. Trumpf, T. Hamel, and T. Cheviron. Complementary filter design on the special euclidean group SE (3). In Proc. of the 2007 European Control Conference, 2007.

[5] J.A. Castellanos, J. Neira, and J.D. Tardós. Limits to the consistency of EKF-based SLAM. IFAC Symposium on Intelligent Autonomous Vehicles, 2004.

[6] JA Castellanos, R. Martinez-Cantin, JD Tardós, and J. Neira. Robocentric map joining: Improving the consistency of EKF-SLAM. Robotics and Autonomous Systems, 55(1):21-29, 2007.

[7] G. Chirikjian. Stochastic Models, Information Theory, and Lie Groups, Volume 2. 2012.

[8] H. Durrant Whyte and T. Bailey. Simultaneous localisation and mapping (SLAM): Part I the essential algorithms. IEEE Robotics and Automation Magazine, 13(2):99-110, 2006.

[9] E. B. Dynkin. Calculation of the coefficients in the Campbell-Hausdorff formula. Doklady Akademii Nauk SSSR, 57:323-326, 1947.

[10] E. Eade and T. Drummond. Scalable monocular SLAM. In IEEE Conf. on Computer Vision and Pattern Recognition (CVPR), volume 1, pages 469-476, 2006.

[11] D. Hahnel, W. Burgard, D. Fox, and S. Thrun. An efficient FastSLAM algorithm for generating maps of large-scale cyclic environments from raw laser range measurements. In Proc. of IEEE Int'l Conf. on Intelligent Robots and Systems (IROS), volume 1, pages 206-211, 2003.

[12] D.J. Higham. An algorithmic introduction to numerical simulation of stochastic differential equations. SIAM review, pages 525-546, 2001.

[13] S. Huang and G. Dissanayake. Convergence and consistency analysis for extended Kalman filter based SLAM. IEEE Trans. on Robotics, 23(5):1036-1049, 2007.

[14] S.J. Julier and J.K. Uhlmann. A counter example to the theory of simultaneous localization and map building. In IEEE Int'l Conf. on Robotics and Automation (ICRA), volume 4, pages 4238-4243, 2001.
[15] J. Kwon and K.M. Lee. Monocular SLAM with locally planar landmarks via geometric Rao-Blackwellized particle filtering on Lie groups. In IEEE Conf. on Computer Vision and Pattern Recognition (CVPR), pages 15221529, 2010.

[16] M. Montemerlo, S. Thrun, D. Koller, and B. Wegbreit. FastSLAM: A factored solution to the simultaneous localization and mapping problem. In Proc. of the Nat. Conf. on Artificial Intelligence, pages 593-598, 2002.

[17] M. Montemerlo, S. Thrun, D. Koller, and B. Wegbreit. FastSLAM 2.0: An improved particle filtering algorithm for simultaneous localization and mapping that provably converges. In Int'l Joint Conf. on Artificial Intelligence, volume 18, pages 1151-1156. Lawrence Erlbaum Associates LTD, 2003.

[18] K. Murphy. Bayesian map learning in dynamic environments. Advances in Neural Information Processing Systems (NIPS), 12:1015-1021, 1999.

[19] W. Park, Y. Wang, and G.S. Chirikjian. The path-ofprobability algorithm for steering and feedback control of flexible needles. The Int'l J. of Robotics Res., 29(7): 813-830, 2010.

[20] G. Silveira, E. Malis, and P. Rives. An efficient direct approach to visual SLAM. IEEE Trans. on Robotics, 24 (5):969-979, 2008.

[21] R. Smith, M. Self, and P. Cheeseman. Estimating uncertain spatial relationships in robotics. Autonomous robot vehicles, 1:167-193, 1990.

[22] C. Stachniss, G. Grisetti, W. Burgard, and N. Roy. Analyzing gaussian proposal distributions for mapping with Rao-Blackwellized particle filters. In Proc. of IEEE Int'l Conf. on Intelligent Robots and Systems (IROS), pages 3485-3490, 2007.

[23] S. Thrun, W. Burgard, and D. Fox. A real-time algorithm for mobile robot mapping with applications to multirobot and 3D mapping. In IEEE Int'l Conf. on Robotics and Automation (ICRA), volume 1, pages 321-328, 2000.

[24] S. Thrun, W. Burgard, and D. Fox. Probabilistic robotics. MIT Press, 2006.

[25] Y. Wang and G. S. Chirikjian. Nonparametric secondorder theory of error propagation on motion groups. The Int'l J. of Robotics Res., 27(11-12):1258-1273, 2008.

[26] Y. Zhou and G.S. Chirikjian. Probabilistic models of dead-reckoning error in nonholonomic mobile robots. In IEEE Int'l Conf. on Robotics and Automation (ICRA), volume 2, pages 1594-1599, 2003. 